\title{
MATHEMATICAL MODEL TO TAKE INTO ACCOUNT THE INFLUENCE OF SATURATION OF THE MAGNETIC CIRCUIT ON THE STARTING CHARACTERISTICS OF A SYNCHRONOUS MOTOR
}

\author{
Olimjon Toirov ${ }^{1}$, Kamoliddin Alimkhodjaev ${ }^{1}$, Nurali Pirmatov $^{1}$, Aziza Kholbutaeva $^{1}$ \\ ${ }^{1}$ Tashkent State Technical University, Department of Electrical machine, Tashkent, 100095, Uzbekistan
}

\begin{abstract}
The results of the development of a mathematical model of a synchronous motor and a study of the influence of the degree of saturation of the magnetic circuit on the transient processes of direct start-up are presented. It is noted that the existing methods of research and calculation of transient processes of synchronous machines are based on the application of the superposition (superposition) method, which excludes their use in nonlinear systems.
\end{abstract}

\section{Introduction}

The reliable operation of large electric power systems largely depends on the reliable operation of synchronous machines that are used in all power plants, large industrial enterprises of the national economy, pumping stations for water and agriculture [4-5]. Their troublefree operation largely depends on the correct calculation of parameters during design, therefore, at the present stage of development of technology and technology, high requirements are imposed on the accuracy of calculating the parameters and characteristics of electrical machines.

It is known that in the general theory of electrical machines, in order to simplify mathematical expressions and the processes of their calculation, a number of generally accepted assumptions are adopted, which allow instead of a real electrical machine to consider some of its linearized model. Such idealized electrical machines differ from real machines in the following simplifications. There is no saturation of the magnetic circuit; the air gap is smooth, i.e. the toothed surfaces of the rotor and stator are replaced with smooth surfaces; there are no hysteresis, eddy currents in steel and current displacement in copper windings; curves of magnetomotive forces and magnetic inductions are assumed to be sinusoidally distributed in space; the inductive resistance of the scattering of the windings does not depend on the position of the rotor; stator windings are located symmetrically [1]. When studying certain modes of idealized electric machines, the components of the magnetic field and the field in each section of the magnetic circuit are considered independently of each other. For example, when studying salient-pole synchronous machines using the method of two reactions, the longitudinal component of the field is considered separately from the transverse component, the magnetic fields from the higher harmonic magnetizing forces are considered independent of the fundamental harmonic field. Such assumptions make it possible to apply the superposition method, which greatly facilitates the calculations of electrical machines.

When designing modern synchronous machines, in contrast to an idealized electric machine, in order to reduce its mass, the operating point is selected on the nonlinear section of the magnetization curve - much higher than the so-called "knee of saturation" [2]. With changes in voltage, frequency and load, the degree of saturation changes, and this affects the performance of the machine. Especially, in studies of transient modes, there is an urgent need to take into account the nature of the behavior in time of machine parameters, which will not be constant with a change in the degree of saturation. In addition, in a real electric machine, the parameters of the windings are not constant. Active resistance depends on a number of factors, such as temperature, changing over time, fluctuations in the load current, changes in the frequency of the current. Reactive resistances depend on the design features of the core of the machine, the degree of saturation of the sections of the magnetic circuit, as well as on the magnitude and nature of the load.

The change in the saturation of the magnetic system of the machine, first of all, affects the changes in the magnitudes of the amplitudes and frequencies of the higher harmonic magnetic flux, current and voltage of the windings. In turn, the higher harmonic magnetic flux affects the differential scattering reactivity of the machine, the magnitude of the electromagnetic moment and the characteristics of the transient mode.

Long-term experience of researchers of electrical machines by scientists from leading schools of electromechanics $[1,2]$ has shown that analytical studies of transient processes with the use of idealization of machines give results that are quite consistent with the 
results of experiments. However, the desire of designers of modern electrical machines to reduce the weight and dimensions by increasing the saturation of the machine and the high growth rate of the use of semiconductor frequency converters for powering synchronous motors lead to the need to conduct research into account of the nonlinearity of the magnetization characteristic [2].

The process of taking into account the influence of saturation of the magnetic circuit of large synchronous motors on the transient start-up process by conducting an experiment is a laborious task and leads to undesirable consequences. Therefore, it is advisable to solve this problem using mathematical models (MM) and comparing the results of calculations with experimental data on physical models of synchronous machines of low power.

The purpose of this work is to develop a mathematical model of a synchronous motor and study with its help the influence of saturation of the magnetic circuit on the characteristics of the transient process of starting up a synchronous motor.

\section{Mathematical models}

The main obstacle to using the existing theory of idealized electrical machines in the development of a mathematical model of transient processes of a synchronous machine, taking into account the saturation of the magnetic system, is the question of applying the superposition method, which is intended only for use in linear systems.

To develop the MM of transient processes for starting a synchronous motor, it is required to write down the balance equations for the voltages of the windings, i.e. differential equations of voltages of phase windings of electric circuits, balance equations of moments, rotor speed and analytical dependence of the electromagnetic moment. The voltage equations for phases $\mathrm{a}, \mathrm{b}$ and $\mathrm{c}$ of the stator windings, excitation and starting winding can be written in the following form:

$$
\begin{aligned}
& u_{a}=-d \Psi_{a} / d t-i_{a} r_{s} ; \\
& u_{b}=-d \Psi_{b} / d t-i_{b} r_{s} ; \\
& u_{c}=-d \Psi_{c} / d t-i_{c} r_{s} ; \\
& u_{\mathrm{B}}=-d \Psi_{\mathrm{B}} / d t-i_{\mathrm{B}} r ; \\
& 0=-d \Psi_{\Pi} / d-i_{\Pi} r_{\Pi},
\end{aligned}
$$

where $\Psi_{a}, \Psi_{b}, \Psi_{c}, \Psi_{\text {в }}$ и $\Psi_{\text {п }}$ - full flux linkages with phase windings $a, b, c$ of the stator, excitation and starting winding; $u_{a}, u_{b}, u_{c}, u_{\mathrm{B}}$ and $i_{a}, i_{b}, i_{c}, i_{\mathrm{B}}, i_{\Pi}-$ instantaneous values of phase voltages and currents of stator windings, excitation and starting winding; rs and $\mathrm{rv}, \mathrm{rp}$ are the active resistances of the phase stator windings, the field winding and the starting winding.

The equilibrium equation of the moments of the engine is written in the following form

$$
M_{c}=M_{\text {эм }}+J \cdot d \omega_{r} / d t,
$$

where $M$ - the mutual inductance between the pairs of stator and rotor windings, determined from experimental data.
When developing a mathematical model of a synchronous motor without taking into account the saturation nonlinearities of the magnetic circuit, as mentioned above, equations (1) of the stator and rotor windings are used with the decomposition of the full flux linkage into separate components. In this case, the coefficients in the equations are constant. Taking into account the nonlinearity of the magnetization characteristics, the coefficients of the variables - the parameters of the machine, will be variable depending on the magnitude of the fluxes, which are determined from the experimental data.

We make assumptions about the absence of losses in steel, which allows us to consider the angle of displacement of the curves of the phase current and the magnetic induction created by it to be zero; the toothed surfaces of the rotor and stator are replaced with smooth surfaces. In the future, the presence of stator and rotor teeth will be taken into account especially, since the presence of teeth changes the shape of the starting characteristic.

We consider that the leakage inductances of the windings are small compared to the total inductance of the windings. Then it can be assumed that the flux linkage with some windings can be divided into separate components. Then the coefficients in front of the components are the inductance of the stator windings $L_{l}(t)$, the excitation $L_{6}(t)$ and the starting winding $L_{n}(t)$, the mutual inductances $M(t)$ between the windings and the leakage inductance of the stator windings $L_{\sigma l}(t)$ and the rotor $L_{\sigma \sigma}(t), L_{\sigma n}(t)$ will vary depending on the instantaneous positions of the windings, i.e. will change over time. The dependence of inductances on time is conditional. In reality, the values of $L$ and $M$ are variable coefficients, depending on the relative position of the stator windings and the rotor, which rotates with different acceleration. In the course of solving the problem, we will introduce the appropriate changes associated with changes in inductances.

The study of the influence of leakage fluxes will be carried out in stages: in the first approximation, we assume that the leakage fluxes are closed in air, the leakage inductances of the stator and rotor do not depend on saturation, and take $L_{\sigma S}$ and $L_{\sigma R}$ constant. Then you can make the assumption that $L_{S}(t), L_{\sigma B}(t)$ and $L_{\sigma I}(t)$ will vary according to the following law [2]:

$L_{S}(t)=M(t)+L_{\sigma S}(t) ; L_{B}(t)=M(t)+L_{\sigma B}(t) ; L_{I I}(t)=M(t)+L_{\sigma I I}(t)$.

To study the static mode of synchronous machines using a mathematical model, in order to simplify the equations and coefficients in front of the variables, the coordinate system $d-q$ is used, in which the magnetic fields of the stator and rotor are relatively stationary $\left(\omega_{r}\right.$ $=\omega_{s}$ ). Since in this case the transient process of a synchronous motor in the starting mode $\left(\omega_{r} \neq \omega_{s}\right)$ is studied, we take the coordinate system $\alpha-\beta$, which is used in the study of asynchronous machines

Then 


$$
\left.\begin{array}{l}
\Psi_{S \alpha}=L_{S}(t) i_{S \alpha}+M(t) i_{r \alpha} ; \\
\Psi_{S \beta}=L_{S}(t) i_{S \beta}+M(t) i_{r \beta} ; \\
\Psi_{B \alpha}=L_{B}(t) i_{B \alpha}+M(t) i_{B \alpha} ; \\
\Psi_{\Pi \alpha}=L_{I I}(t) i_{\Pi \alpha}+M(t) i_{\Pi \alpha}
\end{array}\right\}
$$

In order to simplify the structural diagram with nonlinear inductances $L(t)$ and mutual inductance $M(t)$, we introduce new variables - the instantaneous values of the magnetizing currents along the axes of coordinates $\alpha$ and $\beta$, determined by the sum of the instantaneous values of the stator and rotor currents:

$$
i_{m \alpha}=i_{S \alpha}+i_{R \alpha}, i_{m \beta}=i_{S \beta}+i_{R \beta} .
$$

Then the number of equations of the mathematical model of the transient process (6) for starting a synchronous motor will be equal to six: two equations for the voltage of the stator winding along the $\alpha$ and $\beta$ axes, one equation for the voltage of the excitation winding, the starting winding, the balance of moments on the motor shaft and the dependence of the electromagnetic moment. In this case, the voltage equations of the rotor windings: excitation and starting winding will be written only along the coordinate axis $\alpha$, since they are located at the poles of the machine. The voltages of the short-circuited starting winding and the field winding closed to the additional resistance $R_{д}$ will be equal to zero. The resistance of this circuit will be written as $\left(r_{\Pi}+R_{\text {Д }}\right)$.

Having written down the equation (2) of the equilibrium of the moments in terms of the rotor speed $\omega r$ and including in the system of equations of the engine (1), (3) - (5), we construct a mathematical model in the transient start-up process

$$
\begin{aligned}
& u_{S \alpha}=\left[r_{S}+\frac{d}{d t} L_{\sigma S}(t)\right] i_{S \alpha}+\frac{d}{d t} M(t) i_{m \alpha}, \\
& u_{S \beta}=\left[r_{S}+\frac{d}{d t} L_{\sigma S}(t)\right] i_{S \beta}+\frac{d}{d t} M(t) i_{m \beta}, \\
& 0=\left[r_{\Pi}+\frac{d}{d t} L_{\sigma \Pi}(t)\right] i_{\Pi \alpha}+\frac{d}{d t} M(t) i_{m \alpha}+\omega_{r}\left[L_{\Pi} i_{\Pi \beta}+M(t) i_{m \beta}\right], \\
& 0=\left[r_{B}+\frac{d}{d t} L_{\sigma B}(t)\right] i_{B \alpha}+\frac{d}{d t} M(t) i_{m \alpha}+\omega_{r}\left[L_{B} i_{B \alpha}+M(t) i_{m \alpha}\right], \\
& M_{\ni M}(t)=p M(t)\left[i_{m \alpha} i_{\Pi \alpha}-i_{m \beta} i_{\Pi \alpha}+i_{m \alpha} i_{B \alpha}-i_{m \beta} i_{m \alpha}\right], \\
& \omega_{r}=\frac{p}{J}\left[M_{\ni M}(t)-M_{C}(t)\right],
\end{aligned}
$$

where $p$ - the number of pairs of poles of the machine; $M_{\ni M}$ - the electromagnetic moment, which is generated from the interaction of the currents of the following pairs of windings: the magnetizing current of the stator winding along the $\alpha$ and $\beta$ axes with the currents of the starting winding and the excitation winding along the $\alpha$ axis.

In the system of equations, the magnitudes of the inductances $L_{\sigma S}(t), L_{\sigma B}(t)$ and $L_{\sigma \Gamma}(t)$, as well as the mutual inductances between the stator and rotor windings $M(t)$, are replaced by experimentally taken $L_{\sigma S}(\theta), L_{\sigma B}(\theta), L_{\sigma \Gamma}(\theta)$ and $M(\theta)$. In this case, the mutual $M(\theta)$ inductances between the stator windings and the field winding and between the stator windings $M_{S B}\left(\theta_{S I}\right)$ and the starting winding $M_{S B}\left(\theta_{S I}\right)$ are taken as. Here the values $\theta$ are the angles between the corresponding stator and rotor windings, which are determined by solving the differential equation

$$
\omega_{r}=\frac{d \theta}{d t} .
$$

The influence of the degree of saturation of the magnetic circuit on the starting mode of a synchronous motor is determined by approximating the experimentally measured magnetization characteristics. Studies have shown that the use of the piecewise linear approximation method allows significant errors in the construction of starting characteristics (more than 17$19 \%$ ). The volume of calculations when using parabolic polynomials is not less than when using cubic splines, therefore, cubic splines are adopted in the work, they are more accurate when constructing starting characteristics (error no more than 8-12\%) [3].

To determine the solution of a mathematical model to take into account the influence of saturation of the magnetic circuit on the starting characteristics of a synchronous motor, a solution algorithm was drawn up and programming was performed in $\mathrm{C}++$.

For a preliminary assessment of the results of the study of a synchronous motor on a mathematical model by comparing them with experimental data, the starting characteristics of a synchronous motor of the MCA 72-4 type, with a power of $15 \mathrm{~kW}$, voltage of $400 \mathrm{~V}$. and a rotor speed of $1500 \mathrm{rpm}$ were taken at various degrees of saturation magnetic circuit of the machine.

The influence of the degree of saturation of the magnetic circuit on the starting characteristics of the SM was simulated by changing the mains voltage applied to the stator winding. At the same time, for a given SM, the air gap induction values were taken at a nominal induction $=0.8 \mathrm{~T}$, an overestimated induction of 1.0 and $1.2 \mathrm{~T}$.

\section{Conclusion}

Studies of the mathematical model to take into account the effect of saturation of the magnetic circuit on the starting characteristics of a synchronous motor showed that with an increase in the degree of saturation of the magnetic circuit, the influence of higher harmonic magnetic flux on the electromagnetic torque of a synchronous motor increases, as a result of which its braking character 


\section{References}

1. Kononenko Ye.V., Sipaylov G.A., Xor'kov N.A. Elektricheskie mashini (spesial'niy kurs). Uchebnoe posobie dlya vuzov. - M.: «Visshaya shkola», 1975. 279 s.

2. Kopilov I.P. Elektricheskie mashini. Ucheb. dlya vuzov. 3-e izd., ispr. - M.: Vissh. shk., 2002. - 607 s.

3. Kholbutaeva A.K., Pirmatov N.B. Method of calculating the leakage flux taking into account nonlinear saturation of the magnetic circuit. European Applied Science. N12, 2015. p. 30.

4. Olimjon Toirov, Utkir Mirkhonov and etc. Overview of Compressor Installations and Issues of Their Energy saving // International Journal of Advanced Research in Science, Engineering and Technology. India, 2019.Vol.6, N10. P. 11446-11452.

5. Olimjon Toirov, Utkir Mirkhonov. Principles for Controlling the Excitation of Synchronous Motors of the Compressor Installation // International Journal of Advanced Research in Science, Engineering and Technology. - India, 2020.Vol.7, N5. P. 13876-13881. 